JOURNAL OF

SYMPLECTIC GEOMETRY

Volume 5, Number 4, 357-384, 2007

\title{
OZSVÁTH-SZABÓ INVARIANTS AND TIGHT CONTACT 3-MANIFOLDS, III
}

\author{
Paolo Lisca And András I. Stipsicz
}

\begin{abstract}
We characterize $L$-spaces which are Seifert fibered over the 2-sphere in terms of taut foliations, transverse foliations and transverse contact structures. We give a sufficient condition for certain contact Seifert fibered 3-manifolds with $e_{0}=-1$ to have nonzero contact OzsváthSzabó invariants. This yields an algorithm for deciding whether a given small Seifert fibered $L$-space carries a contact structure with nonvanishing contact Ozsváth-Szabó invariant. As an application, we prove the existence of tight contact structures on some 3-manifolds obtained by integral surgery along a positive torus knot in the 3 -sphere. Finally, we prove planarity of every contact structure on small Seifert fibered $L$-spaces with $e_{0} \geq-1$, and we discuss some consequences.
\end{abstract}

\section{Introduction}

The Ozsváth-Szabó homology groups of a closed, oriented 3-manifold $Y$ $[\mathbf{3 6}, \mathbf{3 7}]$ capture important topological information about $Y$. For example, by [35, Theorem 1.1] the Thurston semi-norm is determined by the evaluation of the first Chern classes of $\operatorname{spin}^{c}$ structures with nontrivial OzsváthSzabó homology groups. For rational homology spheres, however, the Thurston norm is trivial, while the Ozsváth-Szabó homology groups are special: the group $\widehat{\mathrm{HF}}(Y, \mathbf{t})$ has odd rank for each $\operatorname{spin}^{c}$ structure $\mathbf{t} \in \operatorname{Spin}^{c}(Y)$. A rational homology sphere $Y$ shows the simplest possible Heegaard Floertheoretic behavior if for every $\operatorname{spin}^{c}$ structure $\mathbf{t} \in \operatorname{Spin}^{c}(Y)$ the group $\widehat{\mathrm{HF}}(Y, \mathbf{t})$ with integral coefficients is isomorphic to $\mathbb{Z}$, in which case $Y$ is called an $L$-space. In the present paper, we shall always use $\mathbb{Z} / 2 \mathbb{Z}$ coefficients. In this case, if $Y$ is an $L$-space then $\widehat{\mathrm{HF}}(Y, \mathbf{t}) \cong \mathbb{Z} / 2 \mathbb{Z}$ for every $\mathbf{t} \in \operatorname{Spin}^{c}(Y)$. Since $\widehat{\mathrm{HF}}(Y, \mathbf{t}) \cong \widehat{\mathrm{HF}}(-Y, \mathbf{t})$ for each $\mathbf{t} \in \operatorname{Spin}^{c}(Y)$, the 3manifold $Y$ is an $L$-space if and only if $-Y$ is. 
If $Y$ is a 3-manifold with elliptic geometry then $Y$ is an $L$-space [39, Proposition 2.3]. In particular, lens spaces are $L$-spaces. Seifert fibered $L$-spaces can be characterized combinatorially using the results of $[\mathbf{2 8}, \mathbf{3 3}]$.

More generally, by [28] the boundary of a negative definite 4-dimensional plumbing is an $L$-space if it is the link of a rational surface singularity. In [34, Section 5, Question 11], it was asked whether there is a topological characterization of $L$-spaces. In the first part of this paper, we study the topological significance of being an $L$-space for Seifert fibered rational homology 3 -spheres.

It is proved in [35, Theorem 1.4] that an $L$-space admits no taut foliation. Our first result shows that the converse to this statement holds for Seifert fibered rational homology 3 -spheres. In addition, we find that it is equivalent to the nonexistence of a transverse foliation (i.e., a foliation transverse to the fibers of the Seifert fibration), as well as to the nonexistence of positive, transverse contact structures for at least one orientation.

Let $M$ be a closed, oriented rational homology 3 -sphere which carries a Seifert fibration over $S^{2}$. Then, $M$ is orientation preserving diffeomorphic to the oriented 3 -manifold $M\left(e_{0} ; r_{1}, r_{2}, \ldots, r_{k}\right)$ with surgery presentation given by Figure 1 , where $e_{0} \in \mathbb{Z}, r_{i} \in(0,1) \cap \mathbb{Q}$ and $r_{1} \geq r_{2} \geq \cdots \geq r_{k}$.

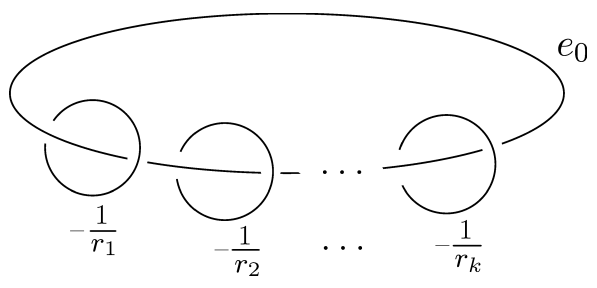

Figure 1. Surgery diagram for $M\left(e_{0} ; r_{1}, r_{2}, \ldots, r_{k}\right)$.

Moreover, the surgery picture shows that $M\left(e_{0} ; r_{1}, \ldots, r_{k}\right)$ carries a natural Seifert fibration over $S^{2}$, which coincides (up to isotopy) with the image of the fibration on $M$ under the above diffeomorphism. Throughout the paper, we always implicitely refer to this natural fibration on $M\left(e_{0} ; r_{1}, \ldots, r_{k}\right)$.

Define

$$
e(M):=e_{0}(M)+r_{1}+r_{2}+\cdots+r_{k} .
$$

Notice that

$$
-M\left(e_{0} ; r_{1}, r_{2}, \ldots, r_{k}\right)=M\left(-e_{0}-k ; 1-r_{1}, 1-r_{2}, \ldots, 1-r_{k}\right),
$$

therefore we have $e(-M)=-e(M)$. It is known that $M$ is a rational homology sphere if and only if $e(M) \neq 0$.

Theorem 1.1. Let us suppose that $M$ is an oriented rational homology 3-sphere which is Seifert fibered over $S^{2}$. Then, the following statements are equivalent: 
(1) $M$ is an L-space;

(2) Either $M$ or $-M$ carries no positive, transverse contact structures;

(3) $M$ carries no transverse foliations;

(4) $M$ carries no taut foliations.

It was proved in $[\mathbf{2 1}]$ that an oriented Seifert fibered rational homology 3 -sphere $M=M\left(e_{0}, r_{1}, r_{2}, \ldots, r_{k}\right)$ with $r_{1} \geq r_{2} \geq \cdots \geq r_{k}$ admits no positive transverse contact structure if and only if

- $e_{0}(M) \geq 0$, or

- $e_{0}(M)=-1$ and there are no relatively prime integers $m>a$ such that

$$
m r_{1}<a<m\left(1-r_{2}\right) \text { and } m r_{i}<1 i=3, \ldots, k .
$$

Combined with this result, Theorem 1.1 gives a simple characterization of $L$-spaces among rational homology 3 -spheres of the form $M\left(e_{0} ; r_{1}, \ldots, r_{k}\right)$.

In the second part of the paper, we give a sufficient condition for certain contact Seifert fibered 3-manifolds with $e_{0}=-1$ to have nonzero contact Ozsváth-Szabó invariants. Combining this result with Theorem 1.1 and with a result of Paolo Ghiggini (Theorem 1.3 below) yields an algorithm for deciding whether an $L$-space of the form $M\left(e_{0} ; r_{1}, r_{2}, r_{3}\right)$ carries a contact structure with nonvanishing contact Ozsváth-Szabó invariant.

Consider the contact surgery diagram of Figure 2. The diagram should be interpreted as representing all possible contact $\left(-\frac{1}{r_{i}}\right)$-surgeries in case these are not unique (see Section 2 for details). With this convention, Figure 2 represents a family of contact structures on the manifold $M=$ $M\left(-1 ; r_{1}, \ldots, r_{k}\right)$. Given an oriented contact structure $\xi$ on $M$, let $\mathbf{t}_{\xi}$ denote the $\operatorname{spin}^{c}$ structure induced by $\xi$ and $d_{3}(\xi) \in \mathbb{Q}$ the 3 -dimensional invariant determined by the homotopy class of the oriented 2-plane field $\xi[\mathbf{1 7}]$. Finally, denote by $d(M, \mathbf{t}) \in \mathbb{Q}$ the $d$-invariant of $(M, \mathbf{t})($ cf. $[\mathbf{3 2}])$.

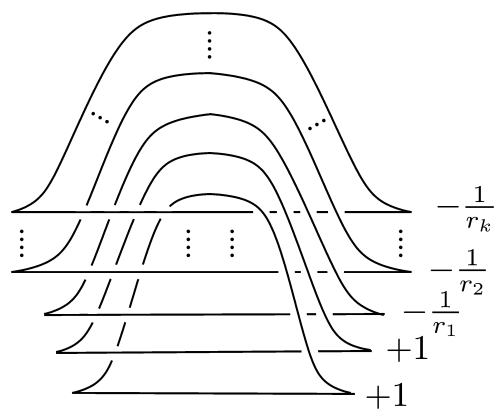

Figure 2. Contact structures on $M\left(-1 ; r_{1}, r_{2}, \ldots, r_{k}\right)$. 
Theorem 1.2. Let $(M, \xi)$ be any of the contact, Seifert fibered 3-manifolds given by Figure 2. Suppose that

$$
e(M)=r_{1}+r_{2}+\cdots+r_{k}-1>0,
$$

and

$$
d_{3}(\xi)=d\left(M, \mathbf{t}_{\xi}\right)
$$

Then the contact invariant $c(M, \xi) \in \widehat{\mathrm{HF}}\left(-M, \mathbf{t}_{\xi}\right)$ is nonzero.

Theorem 1.2 applies to some Seifert fibered 3-manifolds which are not $L$-spaces (such as, e.g., $M\left(-1 ; \frac{5}{12}, \frac{1}{3}, \frac{1}{3}\right)$ ), but it is particularly useful when $k=3$ and the 3 -manifold $M$ is an $L$-space. To explain why, we need a small digression.

Suppose that $(M, \xi)$ is a contact, Seifert fibered 3-manifold. Then, a Legendrian knot in $M$ smoothly isotopic to a regular fiber admits two framings: one coming from the fibration and another one coming from the contact structure $\xi$. The difference between the contact framing and the fibration framing is the twisting number of the Legendrian curve. We say that $\xi$ has maximal twisting equal to zero if there is a Legendrian knot $L$ isotopic to a regular fiber such that $L$ has twisting number zero. According to a result of Ghiggini, if $M=M\left(e_{0} ; r_{1}, r_{2}, r_{3}\right)$ admits no transverse contact structure then each tight contact structure on $M$ has maximal twisting equal to zero.

Theorem 1.3 ([13], Corollary 4.6). $M=M\left(e_{0} ; r_{1}, r_{2}, r_{3}\right)$ admits a tight contact structure with negative twisting if and only if $M$ admits a transverse contact structure.

Thus, combining Theorems 1.1 and 1.3 , we see that if $M=M\left(e_{0} ; r_{1}, r_{2}, r_{3}\right)$ is an L-space, then each tight contact structure $\xi$ on $M$ has maximal twisting equal to zero. On the other hand, in Section 6 (see Proposition 6.1) we show that every tight contact structure with maximal twisting equal to zero on a 3-manifold of the form $M\left(e_{0} ; r_{1}, r_{2}, r_{3}\right)$ is given by one of the diagrams represented by Figure 2. Moreover, the quantities $d_{3}(\xi)$ and $d\left(M, \mathbf{t}_{\xi}\right)$ can be computed algorithmically from a contact surgery presentation. Therefore Theorem 1.2 yields an algorithm for deciding whether an $L$-space of the form $M\left(e_{0} ; r_{1}, r_{2}, r_{3}\right)$ carries a contact structure with nonvanishing contact Ozsváth-Szabó invariants. (Recall that contact structures with nonvanishing contact Ozsváth-Szabó invariant are tight.) In fact, one can view Theorem 1.2 as a useful tool to attack the classification problem for tight contact structures on small Seifert fibered $L$-spaces with $e_{0}=-1$. (Tight contact structures on small Seifert fibered 3-manifolds with $e_{0} \neq-1,-2$ have been classified $[\mathbf{1 4}, \mathbf{4 4}]$, but the classification for $e_{0}=-1,-2$ is expected to be considerably harder, due to the presence of nonfillable structures $[\mathbf{1 1}, \mathbf{2 2}]$ as well as manifolds with no tight contact structures $[\mathbf{1 0}, \mathbf{2 5}]$.)

As an application of Theorem 1.2, we prove the existence of tight contact structures on some of the 3-manifolds obtained by integral surgery along a 
positive torus knot in $S^{3}$. To put this result in perspective, recall that we showed in [24] that for the positive $(p, q)$-torus knot $T_{p, q} \subset S^{3}$ the 3-manifold $S_{r}^{3}\left(T_{p, q}\right)$ obtained by topological $r$-surgery along $T_{p, q}$ carries tight contact structures for every rational $r$ except possibly when $r=p q-p-q$. In this latter case, the idea used in [24] does not work, since it would require the application of contact 0 -surgery, which automatically leads to overtwisted structures. In [25] we analyzed the existence of tight contact structures on the 3-manifold $S_{r}^{3}\left(T_{p, q}\right)$ with $r$ equal to the "critical" surgery coefficient $p q-p-q$. We showed that for $(p, q)=(2,2 n+1)$ the corresponding manifolds do not admit any tight structures (extending an earlier result of Etnyre and Honda [10], who proved the same result for $(p, q)=(2,3))$. On the other hand, in the same paper [25] we verified that $S_{p^{2} n-p n-1}^{3}\left(T_{p, p n+1}\right)$ does carry tight contact structures for every $n \geq 1$ and $p \geq 3$ odd. The parity assumption on $p$ played a crucial role in the argument, since in this case the candidate contact structure induced a spin structure. This allowed us to use a certain $\mathbb{Z} / 2 \mathbb{Z}$ symmetry (the so-called $J$-map) built in Heegaard Floer theory to prove the nonvanishing of the contact invariant. A little computer search using the algorithm outlined above showed that for $p$ even one cannot always find a tight contact structure with nonzero contact Ozsváth-Szabó invariant inducing a spin structure. For example, there is no such structure on $S_{11}^{3}\left(T_{4,5}\right)$. This implies that when $p$ is even, a different approach should be used. In Section 5 we use Theorem 1.2 to prove.

Theorem 1.4. For each $p>2$, the manifold $S_{p^{2}-p-1}^{3}\left(T_{p, p+1}\right)$ carries tight contact structures.

Using the fact that every tight contact structure with zero maximal twisting on a 3-manifold of the form $M\left(e_{0} ; r_{1}, r_{2}, r_{3}\right)$ is given by a surgery diagram as in Figure 2 (for $k=3$ ), we can prove:

Theorem 1.5. A tight contact structure $\xi$ with zero maximal twisting on a small Seifert fibered 3-manifold $M\left(e_{0} ; r_{1}, r_{2}, r_{3}\right)$ is compatible with a planar open book decomposition.

An immediate corollary of Theorem 1.5 is the following characterization of small Seifert fibered $L$-spaces:

Corollary 1.6. A rational homology 3 -sphere $M=M\left(e_{0} ; r_{1}, r_{2}, r_{3}\right)$ is an $L$-space if and only if on either $M$ or $-M$ every contact structure is planar.

Another corollary of Theorem 1.5 is the following.

Corollary 1.7. If $M=M\left(e_{0} ; r_{1}, r_{2}, r_{3}\right)$ with $e_{0} \geq-1$ and $M$ is an $L$-space, then each contact structure on $M$ is planar.

In view of a recent result of Abbas, Cieliebak and Hofer [1] Corollary 1.7 implies: 
Corollary 1.8. If $M=M\left(e_{0} ; r_{1}, r_{2}, r_{3}\right)$ with $e_{0} \geq-1$ and $M$ is an $L$-space, then each contact structure $\xi$ on $M$ satisfies the Weistein conjecture, that is, every Reeb vector field for $(M, \xi)$ admits a periodic orbit.

The paper is organized as follows. In Section 2 we collect some background material. In Sections 3, 4 and 5 we prove, respectively, Theorems 1.1, 1.2 and 1.4. Section 6 is devoted to examining compatible open books and establishing Theorem 1.5 and its corollaries.

\section{Preliminaries}

2.1. Transverse contact structures and transverse foliations. Here we collect some known results on transverse contact structures and foliations on the Seifert fibered 3-manifold

$$
M=M\left(e_{0} ; r_{1}, r_{2}, \ldots, r_{k}\right), \quad r_{1} \geq r_{2} \geq \cdots \geq r_{k}
$$

defined by Figure 1. Consider

$$
\Gamma(M):=\left(r_{1}, r_{2}, \ldots, r_{k}\right) \in(\mathbb{Q} \cap(0,1))^{k} .
$$

We say that $\Gamma(M)$ is realizable if there exist coprime integers $m>a>0$ such that

$$
r_{1}<\frac{a}{m}, r_{2}<\frac{m-a}{m} \text { and } r_{3}, \ldots, r_{k}<\frac{1}{m} .
$$

In Section 3 we shall use the following theorem, obtained by combining results of Jankins-Neumann and Naimi, and stated here in our present notation

Theorem $2.1([\mathbf{2 0}, \mathbf{2 7}])$. Let $M=M\left(e_{0} ; r_{1}, r_{2}, \ldots, r_{k}\right)$ be as above. Then $M$ carries a smooth foliation transverse to the Seifert fibration if and only if one of the following holds:

- $-k+2 \leq e_{0} \leq-2$;

- $e_{0}=-1$ and $\Gamma(M)$ is realizable;

- $e_{0}=-k+1$ and $\Gamma(-M)$ is realizable.

The following theorem is part of a result [21, Theorem 1.3] of the first named author and of Gordana Matić (Ko Honda [19] and Paolo Ghiggini $[\mathbf{1 3}]$ also obtained similar results).

Theorem 2.2 ([21]). Let $M=M\left(e_{0} ; r_{1}, r_{2}, \ldots, r_{k}\right)$ be as above. Then $M$ carries a positive contact structure transverse to the Seifert fibration if and only if one of the following holds:

- $k \leq 2$ and $e(M):=e_{0}+\sum_{i} r_{i}<0$;

- $e_{0} \leq-2$;

- $e_{0}=-1$ and $\Gamma(M)$ is realizable. 
Recall that the Seifert fibered 3 -manifold $M=M\left(e_{0} ; r_{1}, r_{2}, \ldots, r_{k}\right)$ is defined by the surgery diagram of Figure 1. Applying inverse slam-dunks, the diagram can be turned into a surgery diagram involving only integer coefficients: replace each $\left(-\frac{1}{r_{i}}\right)$-surgery with a sequence of integer surgeries along a chain of unknots, where the integral surgery coefficients are given by the coefficients of the continued fraction expansion

$$
-\frac{1}{r_{i}}=a_{0}^{(i)}-\frac{1}{a_{1}^{(i)}-\frac{1}{\ddots \cdot-\frac{1}{a_{k}^{(i)}}}}, \quad a_{j}^{(i)} \leq-2 .
$$

The integral surgery diagram defines a 4 -manifold $W=W\left(e_{0} ; r_{1}, \ldots, r_{k}\right)$. Notice that in the diagram all knots are unknots, they are arranged along a star shaped tree $T$, and all framings are at most -2 except the central one, which is equal to $e_{0}$. The 4-manifold $W$ is endowed with an $S^{1}$-action obtained by equivariant plumbing according to $T$, where each vertex corresponds to a disk bundle over a sphere. By [30, Chapter 2] $M$ is isomorphic (as a 3-manifold with $S^{1}$-action) to the boundary of $W$. Moreover, it follows from the results of [26] that the $S^{1}$-manifold $W$ carries a symplectic form $\omega$ such that $\omega \wedge \omega>0$ and every orbit of the $S^{1}$-action on $\partial W=M$ is tangent to the kernel of $\left.\omega\right|_{\partial W}$. Therefore, if $\xi$ is a transverse contact structure on $M$ then $\omega \mid \xi \neq 0$. Recall that a symplectic filling of a closed contact 3-manifold $(M, \xi)$ is a symplectic 4 -manifold $(X, \Omega)$ such that (i) $X$ is oriented by $\Omega \wedge \Omega$, (ii) $\partial X=M$ as oriented manifolds, and (iii) $\left.\Omega\right|_{\xi} \neq 0$ at every point of $M$. An immediate corollary of this discussion is the following.

Proposition 2.3. $W=W\left(e_{0} ; r_{1}, r_{2}, \ldots, r_{k}\right)$ carries a symplectic form $\omega$ such that if $M=M\left(e_{0} ; r_{1}, r_{2}, \ldots, r_{k}\right)$ as above carries a positive transverse contact structure $\xi$ then $(W, \omega)$ is a symplectic filling of $(M, \xi)$.

It is known $[\mathbf{2 9}]$ that

$$
b_{2}^{+}(W)= \begin{cases}1 & \text { if } e(M)>0 \\ 0 & \text { if } e(M)<0\end{cases}
$$

The following result is not explicitely stated in [21], but it is implicitely contained in the proof of [21, Theorem 1.3] given in [21, Section 4].

Theorem 2.4 ([21]). Let $M=M\left(e_{0} ; r_{1}, r_{2}, \ldots, r_{k}\right)$ be as above. If $M$ carries no positive contact structure transverse to the Seifert fibration then there exists an oriented surface $\Sigma$ smoothly embedded in $W$ such that $g(\Sigma)>0$ and

$$
\Sigma \cdot \Sigma>2 g(\Sigma)-2 \text {. }
$$


2.2. Contact surgery. Suppose that $L \subset(Y, \xi)$ is a Legendrian knot, that is, a knot tangent to the 2-plane field $\xi$. Oriented normals to $\xi$ along $L$ provide a framing for $L$, called the contact framing. Let $Y_{r}(L)$ denote the result of $r$-surgery along $L$, where the surgery coefficient is measured with respect to the contact framing. Contact structures on $Y_{r}(L)$ can be defined by taking the restriction $\left.\xi\right|_{Y-\nu L}$ of $\xi$ to the complement of a standard convex neighborhood of $L$, and extending it to a contact structure on $Y_{r}(L)$ which is tight on the glued-up solid torus. By the classification of tight contact structures on $S^{1} \times D^{2}[\mathbf{1 6}, \mathbf{1 8}]$, such an extension exists if and only if $r \neq 0$ and is unique if and only if $r=\frac{1}{k}$ for some $k \in \mathbb{Z}$. In general, there are many tight structures on $S^{1} \times D^{2}$ satisfying the same boundary condition. As Honda showed, a tight contact $\left(S^{1} \times D^{2}, \eta\right)$ can be decomposed as

$$
\left(S^{1} \times D^{2}, \eta\right)=\bigcup_{i=1}^{t}\left(T^{2} \times I, \eta_{i}\right) \cup\left(S^{1} \times D^{2}, \eta_{0}\right),
$$

where $\left(S^{1} \times D^{2}, \eta_{0}\right)$ is the standard neighborhood of a Legendrian knot isotopic to $S^{1} \times 0 \subset S^{1} \times D^{2}$ and each $\left(T^{2} \times I, \eta_{i}\right)$ is a "continued fraction block." A continued fraction block $\left(T^{2} \times I, \eta_{i}\right)$ admits a further slicing

$$
\left(T^{2} \times I, \eta_{i}\right)=\bigcup_{j=1}^{s_{i}}\left(T^{2} \times I, \eta_{i}(j)\right),
$$

where each $\left(T^{2} \times I, \eta_{i}(j)\right)$ is a "basic slice" (see [18] for definitions). Basic slices are characterized by a sign + or - . The continued fraction blockdecomposition of $\left(S^{1} \times D^{2}, \eta\right)$ is dictated by the boundary value (the "slope" of $\left.T^{2}=\partial\left(S^{1} \times D^{2}\right)\right)$, which in the case of surgery is determined by the surgery coefficient $r$. The signs of the basic slices, however, rely on choices, giving rise to possibly many tight contact structures on $S^{1} \times D^{2}$ with identical boundary condition.

Ding and Geiges [3] showed how to realize each basic slice decomposition of $\left(S^{1} \times D^{2}, \eta\right)$ by a contact surgery diagram (cf. also [4]). Let $r=\frac{p}{q}$ and if $r>0$ choose $k \in \mathbb{N}$ minimal such that $r^{\prime}=p /(q-k p)<0$. Consider $k$ Legendrian push-offs $L_{1}, \ldots, L_{k}$ of $L$ and perform contact $(+1)$-surgeries along these push-offs. (In this part of the procedure there is no choice.) Next, do contact $r^{\prime}$-surgery along $L$. Suppose that the contact solid torus $\left(S^{1} \times D^{2}, \eta\right)$ to be used in the surgery has basic slice decomposition

$$
\bigcup_{i=1}^{t} \bigcup_{j=1}^{s_{i}}\left(T^{2} \times I, \eta_{i}(j)\right) \cup\left(S^{1} \times D^{2}, \eta_{0}\right) .
$$


After fixing an orientation for $L$, apply a right stabilizations to $L$ for each positive basic slice in

$$
\left(T^{2} \times I, \eta_{1}\right)=\bigcup_{j=1}^{s_{1}}\left(T^{2} \times I, \eta_{1}(j)\right)
$$

and a negative stabilization for each negative basic slice. Denote the result by $L(1)$. Consider a Legendrian push-off $L^{\prime}(1)=L(2)$ of $L(1)$ and perform contact $(-1)$-surgery along $L(1)$. Repeat the above procedure for $L(2)$, using the second continued fraction block of the decomposition. After $t$ steps the procedure terminates providing a Legendrian link in $(Y, \xi)$ along which the prescribed contact $( \pm 1)$-surgeries give the contact structure on $Y_{r}(L)$ obtained by applying contact $r$-surgery with the prescribed extension $\left(S^{1} \times D^{2}, \eta\right)$ on the glued-up solid torus.

2.3. Ozsváth-Szabó homologies. In the seminal papers $[\mathbf{3 6}, 37]$ a collection of homology groups - the Ozsváth-Szabó homologies - $\widehat{\mathrm{HF}}(Y, \mathbf{t})$, $\operatorname{HF}^{ \pm}(Y, t)$ and $\operatorname{HF}^{\infty}(Y, \mathbf{t})$ have been assigned to any closed, oriented $\operatorname{spin}^{c}$ 3 -manifold $(Y, \mathbf{t})$. For simplicity, throughout this paper we shall use $\mathbb{Z} / 2 \mathbb{Z}$ coefficients. The groups in question admit a relative $\mathbb{Z} / d(\mathbf{t}) \mathbb{Z}$-grading, where $d(\mathbf{t})$ is the divisibility of the first Chern class $c_{1}(\mathbf{t})$. When $c_{1}(\mathbf{t})$ is torsion, then $d(\mathbf{t})=0$, and the relative $\mathbb{Z}$-grading lifts to an absolute $\mathbb{Q}$-grading. The groups $\operatorname{HF}^{ \pm}(Y, \mathbf{t})$ and $\operatorname{HF}^{\infty}(Y, \mathbf{t})$ admit $\mathbb{Z}[U]$-module structures, and multiplication by $U$ decreases grading by 2 .

A $\operatorname{spin}^{c}$ cobordism $(W, \mathbf{s})$ between $\operatorname{spin}^{c} 3$-manifolds $\left(Y_{1}, \mathbf{t}_{1}\right)$ and $\left(Y_{2}, \mathbf{t}_{2}\right)$ induces a $\mathbb{Z}[U]$-equivariant homomorphism $F_{W, \mathbf{s}}$ between the corresponding groups, and if $\mathbf{t}_{1}, \mathbf{t}_{2}$ are both torsion $\operatorname{spin}^{c}$ structures then $F_{W, \mathbf{s}}$ shifts degree by

$$
\frac{1}{4}\left(c_{1}^{2}(\mathbf{s})-3 \sigma(W)-2 \chi(W)\right) .
$$

The direct sum of Ozsváth-Szabó homology groups for all spin ${ }^{c}$ structures is usually denoted by $\widehat{\mathrm{HF}}(Y)$ (respectively, by $\mathrm{HF}^{ \pm}(Y), \mathrm{HF}^{\infty}(Y)$ ), while the sum of the maps induced by $(W, \mathbf{s})$ for all $\mathbf{s} \in \operatorname{Spin}^{c}(W)$ is denoted by $F_{W}$ (respectively, $F_{W}^{ \pm}, F_{W}^{\infty}$ ).

A rational homology sphere $Y$ is called an $L$-space if $\widehat{\mathrm{HF}}(Y, \mathbf{t})$ is isomorphic to $\mathbb{Z} / 2 \mathbb{Z}$ for all $\operatorname{spin}^{c}$ structure $\mathbf{t} \in \operatorname{Spin}^{c}(Y)$. Equivalently, the dimension $\operatorname{dim}_{\mathbb{Z} / 2 \mathbb{Z}} \widehat{\mathrm{HF}}(Y)$ is equal to the order $\left|H_{1}(Y ; \mathbb{Z})\right|$. Other equivalent ways to define $L$-spaces is to require that the $U$-action on $\mathrm{HF}^{+}(Y, \mathbf{t})$ is surjective, or that the natural map $\operatorname{HF}^{\infty}(Y, \mathbf{t}) \rightarrow \operatorname{HF}^{+}(Y, \mathbf{t})$ is onto. Yet another characterization is that for an $L$-space $Y$ the group $\widehat{\mathrm{HF}}(Y, \mathbf{t})$ can be identified with the kernel of the $U$-map

$$
U: \mathrm{HF}^{+}(Y, \mathbf{t}) \rightarrow \mathrm{HF}^{+}(Y, \mathbf{t}) .
$$


(The equivalences follow from the long exact sequences connecting the various groups, see [37].) For a rational homology 3-sphere $Y$ and $\operatorname{spin}^{c}$ structure $\mathbf{t} \in \operatorname{Spin}^{c}(Y)$ let the $d$-invariant $d(Y, \mathbf{t})$ of $(Y, \mathbf{t})$ be defined as the absolute degree of the unique nontrivial element $x \in \operatorname{HF}^{+}(Y, \mathbf{t})$ which is in the image of the natural map $\operatorname{HF}^{\infty}(Y, \mathbf{t}) \rightarrow \operatorname{HF}^{+}(Y, \mathbf{t})$ and $U x=0$. It is easy to see that for an $L$-space $Y$ the absolute degree of the generator of $\widehat{\mathrm{HF}}(Y, \mathbf{t})$ is equal to $d(Y, \mathbf{t})$. It is known that $d(-Y, \mathbf{t})=-d(Y, \mathbf{t})$.

Suppose that the manifold $Y(K)$ is given as integral surgery along a knot $K$ in $Y$, while $Y_{1}(K)$ is defined by an integral surgery along $K \subset Y$ with framing one higher. According to [37, Theorem 9.16] the groups corresponding to these 3 -manifolds (together with the maps induced by appropriate cobordisms $W_{1}, W_{2}, W_{3}$ between the 3 -manifolds) fit into the exact triangle:

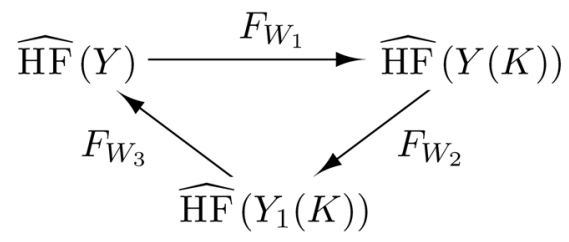

Ozsváth-Szabó homologies are quite hard to compute in general, but for 3-manifolds which can be presented as boundaries of plumbings along negative definite plumbing trees with no "bad" vertices (cf. [33]), such computation has been carried out in [33]. This immediately implies, for example, that a Seifert fibered rational homology 3 -sphere with $k$ multiple fibers and $e_{0}(M) \leq-k$ is an $L$-space, since it can be presented as the boundary of a plumbing tree without "bad" vertices. Since $e_{0}(-M)=-k-e_{0}(M)$ for Seifert fibered spaces, the fact $\operatorname{dim} \widehat{\mathrm{HF}}(Y)=\operatorname{dim} \widehat{\mathrm{HF}}(-Y)$ implies that a Seifert fibered 3-manifold $M=M\left(e_{0} ; r_{1}, r_{2}, \ldots, r_{k}\right)$ with $e_{0}(M) \leq-k$ or $e_{0}(M) \geq 0$ is an $L$-space.

The following two lemmas can be deduced from the results of [33]. They will be used in Section 3 .

Lemma 2.5. Suppose that $W$ is a negative definite cobordism between the L-space $Y_{1}$ and the rational homology 3-sphere $Y_{2}$ obtained by attaching 2handles to $Y_{1}$. Let $\mathbf{s} \in \operatorname{Spin}^{c}(W)$ be a $\operatorname{spin}^{c}$ structure such that the degree shift of the maps

$$
F_{W, \mathbf{s}}: \widehat{\mathrm{HF}}\left(Y_{1},\left.\mathbf{s}\right|_{Y_{1}}\right) \rightarrow \widehat{\mathrm{HF}}\left(Y_{2},\left.\mathbf{s}\right|_{Y_{2}}\right)
$$

and

$$
F_{W, \mathbf{s}}^{+}: \operatorname{HF}^{+}\left(Y_{1},\left.\mathbf{s}\right|_{Y_{1}}\right) \rightarrow \operatorname{HF}^{+}\left(Y_{2},\left.\mathbf{s}\right|_{Y_{2}}\right)
$$

is

$$
d\left(Y_{2},\left.\mathbf{s}\right|_{Y_{2}}\right)-d\left(Y_{1},\left.\mathbf{s}\right|_{Y_{1}}\right) .
$$

Then the maps $F_{W, \mathbf{s}}$ and $F_{W, \mathbf{s}}^{+}$are injective. 
Proof. Since $W$ is negative definite, successive applications of [32, Proposition 9.4] imply that the map

$$
F_{W, \mathbf{s}}^{\infty}: \operatorname{HF}^{\infty}\left(Y_{1},\left.\mathbf{s}\right|_{Y_{1}}\right) \rightarrow \operatorname{HF}^{\infty}\left(Y_{2},\left.\mathbf{s}\right|_{Y_{2}}\right)
$$

is an isomorphism for any $\operatorname{spin}^{c}$ structure. This implies that the map $F_{W, \mathbf{s}}^{+}$is injective provided its degree shift is equal to the difference of the $d$-invariants. The long exact sequence connecting $\widehat{\mathrm{HF}}(Y, \mathbf{t})$ and $\operatorname{HF}^{+}(Y, \mathbf{t})$ together with the fact that the natural map

$$
\widehat{\mathrm{HF}}\left(Y_{1},\left.\mathbf{s}\right|_{Y_{1}}\right) \rightarrow \operatorname{HF}^{+}\left(Y_{1},\left.\mathbf{s}\right|_{Y_{1}}\right)
$$

is injective for the $L$-space $Y_{1}$ imply that if $F_{W, \mathbf{s}}^{+}$is injective then so is the map $F_{W, \mathrm{~s}}$ between the $\widehat{\mathrm{HF}}$-groups, concluding the proof.

Lemma 2.6. Suppose that $W$ is the cobordism from $S^{3}$ to an $L$-space $L$ given by a negative definite plumbing with no "bad" vertex in the sense of [33]. Then for any $\operatorname{spin}^{c}$ structure $\mathbf{t} \in \operatorname{Spin}^{c}(L)$ there is a $\operatorname{spin}^{c}$ structure $\mathbf{s} \in \operatorname{Spin}^{c}(W)$ such that the map

$$
F_{W, \mathbf{s}}: \widehat{\mathrm{HF}}\left(S^{3}\right) \rightarrow \widehat{\mathrm{HF}}(L, \mathbf{t})
$$

is an isomorphism.

Proof. The proof is straightforward using the algorithm of [33] which computes the kernel of the $U$-map on $\operatorname{HF}^{+}(L, \mathbf{t})$. Take the negative definite plumbing $W$ for $L$ and choose the characteristic vector $K \in H^{2}(W ; \mathbb{Z})$ with the following three properties:

- $K$ satisfies the starting condition given in the first paragraph of $[\mathbf{3 3}$, Section 3.1],

- $K$ leads to a final vector with the property given in (16) of the same paragraph, and

- $K$ induces the $\operatorname{spin}^{c}$ structure $\mathbf{t}$ on $L$.

Since the algorithm computes the Ozsváth-Szabó homology of the boundary $L$-space $L$ with the given $\operatorname{spin}^{c}$ structure, such a vector clearly exists. By the formula for the grading given in [33], the final vector of this process defines a $\operatorname{spin}^{c}$ structure on the 4-manifold $W$ satisfying the conditions of Lemma 2.5. Since by assumption $L$ is an $L$-space, this clearly suffices to prove the statement.

2.4. Contact Ozsváth-Szabó invariants. A contact structure $\xi$ on $Y$ determines an element $c(Y, \xi) \in \widehat{\mathrm{HF}}\left(-Y, \mathbf{t}_{\xi}\right)$ which has the following crucial properties [38]:

- $c(Y, \xi)$ is an isotopy invariant of the contact 3-manifold $(Y, \xi)$;

- $c(Y, \xi)=0$ if the contact structure $\xi$ is overtwisted;

- $c(Y, \xi) \neq 0$ if $(Y, \xi)$ is Stein fillable; 
- if $c_{1}\left(\mathbf{t}_{\xi}\right)$ is a torsion class and $c(Y, \xi) \neq 0$ then it is of degree $-d_{3}(\xi)$ in $\widehat{\mathrm{HF}}\left(-Y, \mathbf{t}_{\xi}\right)$, where $d_{3}(\xi) \in \mathbb{Q}$ is the Hopf invariant of the 2-plane field underlying $\xi$.

\section{The proof of Theorem 1.1}

Some of the implications among the equivalent statements of Theorem 1.1 are relatively easy to prove (and have been partly established in the literature), so we will start with those.

$(1) \Longrightarrow(2)$ Suppose that $M$ carries transverse contact structures with both of its orientations. Since $e(-M)=-e(M)$, we can choose the orientation for which the 4-manifold $W$ given by the integer plumbing representation of $M$ has positive $b_{2}^{+}$-invariant. According to Proposition 2.3 this manifold carries a symplectic structure which provides a symplectic filling for any transverse contact structure. Since $b_{2}^{+}(W)>0$, by [35, Theorem 1.4] $M$ cannot be an $L$-space.

(2) $\Longrightarrow(3)$ Since $M \neq S^{1} \times S^{2}$, according to [6] a transverse foliation can be $C^{0}$-approximated by positive as well as negative transverse contact structures. Therefore, the nonexistence of positive transverse contact structures with one orientation prevents the existence of a transverse foliation on $M$.

$(1) \Longrightarrow(4)$ This is part of [35, Theorem 1.4].

(4) $\Longrightarrow(3)$ Since transverse foliations are taut, this implication is trivial.

$(3) \Longrightarrow(2)$ Suppose that $M=M\left(e_{0} ; r_{1}, r_{2}, \ldots, r_{k}\right)$ admits no transverse foliation. Since $M$ is a rational homology sphere, either $e(M)>0$ or $e(-M)=-e(M)>0$. Therefore, if $k \leq 2$ the conclusion follows from Theorem 2.2. Now suppose that $k \geq 3$. According to Theorem 2.1, up to changing the orientation of $M$ we may assume that either $e_{0}(M) \geq 0$ or $e_{0}(M)=-1$ and $\Gamma(M)$ is not realizable. In both cases, by Theorem 2.2, $M$ carries no positive, transverse contact structures.

In the light of our observations above, to complete the proof of the equivalences we only need to show that (2) implies (1), that is, if the Seifert fibered rational homology 3 -sphere $M$ carries no transverse contact structures with one of its orientations then it is an $L$-space. Observe that if $k \leq 2$ then $M$ is a lens space, hence an $L$-space, therefore in this case there is nothing to prove. Likewise, if either $e_{0}(M) \leq-k$ or $e_{0}(M) \geq 0$, then $M$ is an $L$-space. Moreover, if $-k+1 \leq e_{0}(M) \leq-2$ then by Theorem $2.2 M$ carries transverse contact structures. Therefore, up to changing the orientation of $M$ we may assume that $e_{0}(M)=-1$, so to establish Theorem 1.1 it will suffice to prove the following.

Proposition 3.1. If the rational homology 3 -sphere $M=M\left(-1 ; r_{1}, \ldots, r_{k}\right)$ carries no transverse, positive contact structures, then $M$ is an $L$-space. 
Before proving Proposition 3.1 we need some preliminaries. Suppose that $M$ does not carry a transverse positive contact structure. By Theorem 2.4, this implies that the associated 4-manifold $W$ contains a surface $\Sigma$ with $g(\Sigma)>0$ and $\Sigma \cdot \Sigma>2 g(\Sigma)-2$. Let $W_{0}$ be the 4-manifold obtained from $W$ by deleting an open 4-ball from its interior. Then, by [24, Proposition 2.1] the map

$$
F_{W_{0}, \mathbf{s}}: \widehat{\mathrm{HF}}\left(S^{3}\right) \longrightarrow \widehat{\mathrm{HF}}(M)
$$

vanishes for every $\operatorname{spin}^{c}$ structure $\mathbf{s} \in \operatorname{Spin}^{c}\left(W_{0}\right)$. Now let us consider the subcobordism $W_{1}$ obtained by attaching to $S^{3}$ the 2-handles corresponding to the vertices of the plumbing tree with framings at most -2 . This $4-$ manifold is a cobordism between $S^{3}$ and a connected sum of $k$ lens spaces $L$. By standard properties of lens spaces and connected sums, $L$ is an $L$-space. Therefore, $W$ satisfies the assumptions of Lemma 2.6. The attachment of the (-1)-framed circle induces a cobordism $W_{2}$ from $-L$ to $M$. Since $W_{1}$ is negative definite and $W_{0}=W_{1} \cup W_{2}$ is not (because it contains a surface with positive self-intersection), we conclude that $b_{2}^{+}\left(W_{2}\right)=1$.

Lemma 3.2. For each $\operatorname{spin}^{c}$ structure $\mathbf{s}_{2} \in \operatorname{Spin}^{c}\left(W_{2}\right)$ the map $F_{W_{2}, \mathbf{s}_{2}}$ on $\widehat{\mathrm{HF}}(L)$ is zero.

Proof. Let $x \in \widehat{\mathrm{HF}}(L, \mathbf{t})$ be a given generator and consider $\mathbf{s}_{1} \in \operatorname{Spin}^{c}\left(W_{1}\right)$ provided by Lemma 2.6 with the property that $F_{W_{1}, \mathbf{s}_{1}}(g)=x$ for the generator $g \in \widehat{\mathrm{HF}}\left(S^{3}\right)$. By the composition law [40, Theorem 3.4] we have

$$
F_{W_{2}, \mathbf{s}_{2}}(x)=\left(F_{W_{2}, \mathbf{s}_{2}} \circ F_{W_{1}, \mathbf{s}_{1}}\right)(g)=\left(\sum_{\mathbf{t} \in \mathfrak{S}} F_{W_{0}, \mathbf{t}}\right)(g)=0,
$$

where $\mathfrak{S}=\left\{\mathbf{t} \in \operatorname{Spin}^{c}\left(W_{0}\right)|\mathbf{t}|_{W_{i}}=\mathbf{s}_{i}, i=1,2\right\}$. Since $F_{W_{0}, \mathbf{t}}=0$ for all $\mathbf{t} \in \operatorname{Spin}^{c}\left(W_{0}\right)$, this implies $F_{W_{2}, \mathbf{s}_{2}}=0$.

Proof of Proposition 3.1. To verify that $M$ is an $L$-space, consider the surgery exact triangle induced by the cobordism $W_{2}$ :

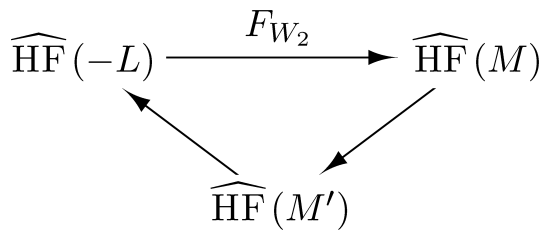

Notice that $M^{\prime}$ is a small Seifert fibered space with $e_{0}\left(M^{\prime}\right)=0$, and hence $M^{\prime}$ is an $L$-space. Since $F_{W_{2}}=0$, we have

$$
\widehat{\mathrm{HF}}\left(M^{\prime}\right)=\widehat{\mathrm{HF}}(L) \oplus \widehat{\mathrm{HF}}(M) .
$$


According to [29, Theorem 8.1], if $Y=M\left(e_{0} ; r_{1}, r_{2}, \ldots, r_{k}\right)$ with with $r_{i}=$ $\frac{p_{i}}{q_{i}}, i=1,2, \ldots, k$ and $e(Y) \neq 0$, then the order of $H_{1}(Y ; \mathbb{Z})$ is equal to

$$
q_{1} q_{2} \cdots q_{k}|e(Y)| \text {. }
$$

Therefore, since $e(M)>0$,

$$
\begin{aligned}
\left|H_{1}(M ; \mathbb{Z})\right| & =\left(\prod_{i=1}^{k} q_{i}\right)\left(\sum_{i=1}^{k} \frac{p_{i}}{q_{i}}-1\right)=\sum_{i=1}^{k} p_{i} \prod_{j \neq i} q_{j}-\prod_{i=1}^{k} q_{i} \\
& =\left|H_{1}\left(M^{\prime} ; \mathbb{Z}\right)\right|-\left|H_{1}(L ; \mathbb{Z})\right| .
\end{aligned}
$$

Thus, we obtain

$$
\operatorname{dim} \widehat{\mathrm{HF}}(M)=\left|H_{1}(M ; \mathbb{Z})\right|
$$

concluding the proof.

\section{The proof of Theorem 1.2}

Let $M=M\left(-1 ; r_{1}, r_{2}, \ldots, r_{k}\right)$ be a 3 -manifold as in the statement of Theorem 1.2, satisfying $e(M)=r_{1}+r_{2}+\cdots+r_{k}-1>0$. Define the family of contact 3-manifolds $\left\{\left(S, \xi_{S}\right)\right\}$ using the contact surgery diagram of Figure 3 with the knot $K_{0}$ deleted.

Lemma 4.1. Each contact 3-manifold $\left(S, \xi_{S}\right)$ is Stein fillable, and each underlying 3-manifold $S$ is an L-space.

Proof. Let $K$ be a Legendrian unknot with maximal Thurston-Bennequin number in the standard contact 3 -sphere. A contact $(+1)$-surgery along $K$ gives a Stein fillable contact 3 -manifold $\left(S^{1} \times S^{2}, \eta\right)$. This follows, e.g., from the fact that the resulting contact structure has nonvanishing OzsváthSzabó invariant [23, Lemma 2.5] and the classification of tight contact structures on $S^{1} \times S^{2}$. Since $\left(S, \xi_{S}\right)$ is given by Legendrian surgery on $\left(S^{1} \times S^{2}, \eta\right)$, $\xi_{S}$ is Stein fillable. Since $e_{0}(M)=-1$, a simple calculation shows that

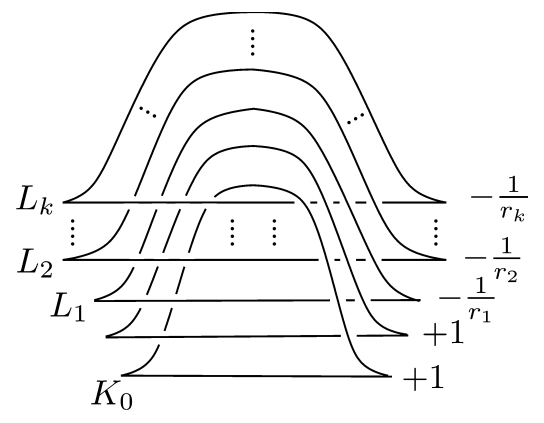

Figure 3. Contact structures on $M$. 
$S$ is a small Seifert fibered 3-manifold with $e_{0}(S)=0$, therefore it is an $L$-space.

Let the 4-manifold $-X$ be defined as the cobordism induced by the contact $(+1)$-surgery along the Legendrian unknot $K_{0}$ in Figure 3. Note that $-X$ is a cobordism between $S$ and $M$. By reversing its orientation, the resulting 4-manifold (a cobordism between $-S$ and $-M$ ) will be denoted by $X$.

Lemma 4.2. The cobordism $X$ is negative definite.

Proof. Converting the contact framings into smooth framings in Figure 3, reversing orientation, blowing up once and applying three Rolfsen twists one easily checks that the cobordism $X$ is given by Figure 4, where the surgery presentation for $-S$ is drawn using solid curves, and the attaching circle of the 2-handle giving $X$ is drawn using the dashed circle. Using a little bit of Kirby calculus, one can easily see that the cobordism $X$ admits an embedding into the 4-manifold

$$
\widehat{W}=W\left(-k+1 ; 1-r_{1}, 1-r_{2}, \ldots, 1-r_{k}\right) \# \overline{\mathbb{C P}^{2}} .
$$

Since

$$
\partial \widehat{W}=M\left(-k+1 ; 1-r_{1}, 1-r_{2}, \ldots, 1-r_{k}\right)=-M
$$

and $e(-M)=-e(M)<0$, we conclude that $W\left(-k+1 ; 1-r_{1}, 1-r_{2}, \ldots\right.$, $\left.1-r_{k}\right)$ is negative definite, and hence so is the blow-up $\widehat{W}$, consequently the cobordism $X \subset \widehat{W}$ is negative definite.

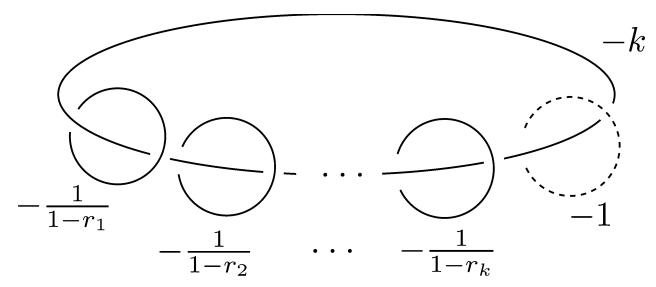

Figure 4. The cobordism $X$.

Proof of Theorem 1.2. Denote by $c^{+}(M, \xi)$ the image of $c(M, \xi)$ under the natural homomorphism $[\mathbf{3 6}, \mathbf{3 7}]$

$$
\widehat{\mathrm{HF}}\left(-M, \mathbf{t}_{\xi}\right) \rightarrow \mathrm{HF}^{+}\left(-M, \mathbf{t}_{\xi}\right) .
$$

Clearly, it is enough to show that $c^{+}(M, \xi) \neq 0$. The contact 3 -manifold $(M, \xi)$ is obtained by a contact $(+1)$-surgery on $\left(S, \xi_{S}\right)$, for some $\left(S, \xi_{S}\right)$ from the family of Lemma 4.1. Therefore, by [12, Lemma 2.11], there is a $\operatorname{spin}^{c}$ structure $\mathbf{s}$ on $X$ such that $F_{X, \mathbf{s}}^{+}\left(c^{+}\left(S, \xi_{S}\right)\right)=c^{+}(M, \xi)$ and

$$
-d_{3}\left(\xi_{S}\right)+\delta(\mathbf{s})=-d_{3}(\xi)
$$


where

$$
\delta(\mathbf{s}):=\frac{1}{4}\left(c_{1}^{2}(\mathbf{s})-3 \sigma(X)-2 \chi(X)\right) .
$$

By assumption, $M$ is a rational homology 3 -sphere, and by Lemma $4.1 S$ is an $L$-space and $\left(S, \xi_{S}\right)$ is Stein fillable. Therefore $c\left(S, \xi_{S}\right) \neq 0$, and it follows that $d\left(-S, \mathbf{t}_{\xi_{S}}\right)=-d_{3}\left(\xi_{S}\right)$. By Lemma $4.2, X$ is negative definite. Moreover, the assumption $d_{3}(\xi)=d\left(M, \mathbf{t}_{\xi}\right)$ immediately implies that the degree shift of the map $F_{X, \mathbf{s}}^{+}$is

$$
\delta(\mathbf{s})=-d_{3}(\xi)+d_{3}\left(\xi_{S}\right)=d\left(-M, \mathbf{t}_{\xi}\right)-d\left(-S, \mathbf{t}_{\xi_{S}}\right) .
$$

Therefore, Lemma 2.5 applies and the map $F_{X, \mathbf{s}}^{+}$is injective, so we conclude that $c^{+}(M, \xi) \neq 0$.

\section{The proof of Theorem 1.4}

Let $K \subset S^{3}$ be a knot, and denote by $S_{r}^{3}(K)$ the 3 -manifold obtained by $r$-surgery along $K$. The proof of Theorem 1.4 will rest on Theorem 1.2, together with Propositions 5.1, 5.2, 5.3 and Lemma 5.4. In the following $M_{p}$ will denote the 3 -manifold $S_{p^{2}-p-1}^{3}\left(T_{p, p+1}\right)$, where $T_{p, p+1}$ is the $(p, p+1)$ torus knot.

Proposition 5.1. Let $\mathbf{t}_{0}$ denote the $\operatorname{spin}^{c}$ structure induced by the (unique) spin structure on the $L$-space $M_{p}$. Then

$$
d\left(M_{p}, \mathbf{t}_{0}\right)= \begin{cases}-\frac{1}{4}(3 p+2) & \text { if } p \text { is even }, \\ -\frac{1}{4}(p+1) & \text { if } p \text { is odd } .\end{cases}
$$

Proof. If $p$ is even then $-M_{p}$ is obtained by plumbing according to a negative definite tree with one bad vertex in the sense of [33], and with $3 p+2$ vertices having all even weights (see, e.g., [25, Figure 4], where $M_{p}=E_{p, 1}$ ).

Therefore, the trivial vector $K=0$ is characteristic and clearly induces $\mathbf{t}_{0}$, so by [33, Corollary 1.5] we have

$$
-d\left(M_{p}, \mathbf{t}_{0}\right)=d\left(-M_{p}, \mathbf{t}_{0}\right)=\frac{K^{2}+3 p+2}{4}=\frac{3 p+2}{4} .
$$

If $p$ is odd, the proof follows easily from [25]. Namely, since $M_{p}=E_{p, 1}$ and $\mathbf{t}_{0}=\mathbf{t}_{E}$ in the notation of [25], by [25, Lemma 6.3, Proposition 6.13] we have

$$
d\left(-M_{p}, \mathbf{t}_{0}\right)=d\left(-L_{p}, \mathbf{t}_{Z}\right)+\frac{1}{4},
$$

where $-L_{p}$ is the oriented boundary of the smooth 4 -manifold $Z_{p}$ given by attaching 2-handles to $B^{4}$ along the framed link of Figure 5 , and $\mathbf{t}_{Z}$ is the $\operatorname{spin}^{c}$ structure induced by the restriction of the unique spin structure on $Z_{p}$. A simple computation gives

$$
d\left(-L_{p}, \mathbf{t}_{Z}\right)=\frac{p}{4},
$$




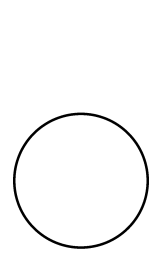

$p+1$

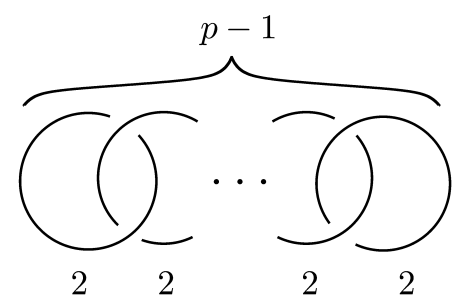

Figure 5. The framed link defining $Z_{p}$.

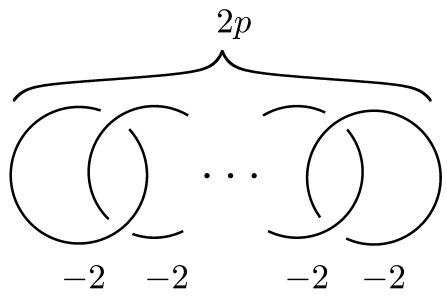

and the conclusion follows.

Let $X_{p}$ denote the 4-manifold obtained by attaching a 2-handle to $D^{4}$ along $T_{p, p+1} \subset S^{3}$ with framing $p^{2}-p-1$. In particular, $\partial X_{p}=M_{p}$. Choose a generator

$$
\left[\Sigma_{p}\right] \in H_{2}\left(X_{p} ; \mathbb{Z}\right) \cong \mathbb{Z}
$$

Since $X_{p}$ is simply connected, a $\operatorname{spin}^{c}$ structure on $X_{p}$ is uniquely determined by its first Chern class, so let $\mathbf{s}_{k} \in \operatorname{Spin}^{c}\left(X_{p}\right)$ denote the $\operatorname{spin}^{c}$ structure with

$$
\left\langle c_{1}\left(\mathbf{s}_{k}\right),\left[\Sigma_{p}\right]\right\rangle=p^{2}-p-1+2 k .
$$

We denote the restriction of $\mathbf{s}_{k}$ to $\partial X_{p}=M_{p}$ by $\mathbf{t}_{k}$. Observe that, in accordance with the notation used in Proposition 5.1, $\mathbf{t}_{0}$ is the $\operatorname{spin}^{c}$ structure induced by the unique spin structure on $M_{p}$. Let

$$
\Delta_{T_{p, p+1}}(t)=a_{0}(p)+\sum_{i=1}^{n_{p}} a_{i}(p)\left(t^{i}+t^{-i}\right)
$$

be the symmetrized Alexander polynomial of the torus knot $T_{p, p+1}$, and define its $j$ th torsion coefficient by

$$
r_{j}(p)=\sum_{i=1}^{n_{p}} i a_{i+|j|}(p) .
$$

Proposition 5.2. For $|k| \leq\left(p^{2}-p-1\right) / 2$, the invariant $d\left(M_{p}, \mathbf{t}_{k}\right)$ is equal to

$$
\frac{\left(\left(p^{2}-p-1\right)-2 j\right)^{2}-\left(p^{2}-p-1\right)}{4\left(p^{2}-p-1\right)}-2 r_{k}(p),
$$

where $j \equiv k \bmod \left(p^{2}-p-1\right)$ and $0 \leq j<p^{2}-p-1$.

Proof. Recall that $S_{p q \pm 1}^{3}\left(T_{p, q}\right)$ are lens spaces. Therefore, combining [32, Theorem 7.2] (together with [32, Remark 7.4]) and [32, Proposition 8.1] (cf. also $[\mathbf{4 1}$, Theorem 1.2]) we have

$$
d\left(M_{p}, \mathbf{t}_{k}\right)-d\left(L\left(p^{2}-p-1,1\right), k\right)=-2 r_{k}(p) .
$$


On the other hand, by $[\mathbf{3 2}$, Section 7$]$

$$
d\left(L\left(p^{2}-p-1,1\right), k\right)=\frac{\left(\left(p^{2}-p-1\right)-2 j\right)^{2}-\left(p^{2}-p-1\right)}{4\left(p^{2}-p-1\right)},
$$

where $j \equiv k \bmod \left(p^{2}-p-1\right)$ and $0 \leq j<p^{2}-p-1$. The statement follows immediately.

Proposition 5.3. The coefficients of the Alexander polynomial $\Delta_{T_{p, p+1}}(t)$ satisfy:

(i) $a_{0}(p)=(-1)^{p+1}$

(ii) all the $a_{i}(p)$ 's are \pm 1 and they alternate in sign, and

(iii) $a_{i}(p)=0$ for $0<i<\frac{p}{2}$.

Proof. It is known (see, for example, [2, Example 9.15]) that

$$
\Delta_{T_{p, p+1}}(t)=\frac{\left(1-t^{p(p+1)}\right)(1-t)}{\left(1-t^{p}\right)\left(1-t^{p+1}\right)} t^{-p(p-1) / 2}
$$

Since

$$
\left(1-t^{p(p+1)}\right)=\left(1-t^{p}\right)\left(1+t^{p}+t^{2 p}+\cdots+t^{p^{2}}\right)
$$

and

$$
\begin{aligned}
(1+ & \left.t^{p}+t^{2 p}+\cdots+t^{p^{2}}\right)(1-t) \\
= & \left(1-t^{p+1}\right)+\left(t^{p}-t^{2 p+1}\right)+\cdots+\left(t^{p^{2}-p}-t^{p^{2}+1}\right)+t^{p^{2}}-t \\
= & \left(1-t^{p+1}\right)\left(1+t^{p}+\cdots+t^{p^{2}-p}\right)+t\left(t^{p^{2}-1}-1\right) \\
= & \left(1-t^{p+1}\right)\left(1+t^{p}+\cdots+t^{p^{2}-p}\right) \\
& -t\left(1-t^{p+1}\right)\left(1+t^{p+1}+t^{2(p+1)}+\cdots+t^{(p+1)(p-2)}\right),
\end{aligned}
$$

we get that the (symmetrized) Alexander polynomial $\Delta_{T_{p, p+1}}$ equals

$$
q(t) t^{-p(p-1) / 2}
$$

where

$$
q(t)=1+t^{p}+\cdots+t^{p^{2}-p}-t\left(1+t^{p+1}+t^{2(p+1)}+\cdots+t^{(p+1)(p-2}\right) .
$$

Therefore, $a_{0}(p)$ is the coefficient of $t^{p(p-1) / 2}$ in $q(t)$, which is equal to 1 if $\frac{1}{2} p(p-1)$ is of the form $i \cdot p$ with $1 \leq i \leq p-1$ (this holds if $p$ is odd), and it is equal to -1 if $\frac{1}{2} p(p-1)$ is of the form $1+k(p+1)$ (which happens with the choice of $k=\frac{1}{2}(p-2)$ for $p$ even). This verifies (i). Part (ii) follows immediately from a close inspection of formula (5.1). To verify (iii), suppose first that $p$ is odd. The first coefficient after $a_{0}(p)$ which is equal to 1 is clearly $a_{p}(p)$, while the first -1 is of index $1+k$ with $k=\frac{1}{2}(p-1)$, showing that $a_{1 / 2(p-1)+1}(p)=a_{1 / 2(p+1)}(p)$ is the first nonzero coefficient after $a_{0}(p)$, verifying (iii) for odd $p$. For $p$ even, a similar argument shows that the 
first coefficient which is equal to -1 is $a_{p+1}(p)$, and the first 1 is $a_{p / 2}(p)$, completing the proof.

Lemma 5.4. For $p>2$ even, we have

$$
d\left(M_{p}, \mathbf{t}_{p / 2}\right)=\frac{\left(p^{2}-2 p-1\right)^{2}}{4\left(p^{2}-p-1\right)}-\frac{1}{4}(p-1)^{2} .
$$

Proof. According to Proposition 5.1 we have

$$
d\left(M_{p}, \mathbf{t}_{0}\right)=-\frac{3 p+2}{4}
$$

and by Proposition 5.2

$$
d\left(M_{p}, \mathbf{t}_{0}\right)=\frac{p^{2}-p-2}{4}-2 r_{0}(p) .
$$

The above identities imply that

$$
r_{0}(p)=\frac{3 p+2}{8}+\frac{p^{2}-p-2}{8}=\frac{p^{2}+2 p}{8} .
$$

On the other hand, by Proposition 5.2

$$
d\left(M_{p}, \mathbf{t}_{p / 2}\right)=\frac{\left(p-\left(p^{2}-p-1\right)\right)^{2}-\left(p^{2}-p-1\right)}{4\left(p^{2}-p-1\right)}-2 r_{p / 2}(p) .
$$

To conclude the proof we will express $r_{p / 2}(p)$ in terms of $r_{0}(p)$. It follows from the definition that

$$
r_{0}(p)=r_{p / 2}(p)+\frac{p}{2} \sum_{i=0}^{n_{p}} a_{i}(p)-\sum_{i=0}^{p / 2}\left(\frac{p}{2}-i\right) a_{i}(p) .
$$

Since by Proposition 5.3, the nonzero coefficients of $\Delta_{T_{p, p+1}}(t)$ alternate in sign and are all \pm 1 , from the assumption that $p$ is even (hence $a_{0}(p)=-1$ ) we get

$$
\sum_{i=0}^{n_{p}} a_{i}(p)=0
$$

On the other hand, by Proposition 5.3 we know that

$$
\sum_{i=0}^{p / 2}\left(\frac{p}{2}-i\right) a_{i}(p)=-\frac{p}{2}
$$

hence

$$
r_{p / 2}(p)=r_{0}(p)-\frac{p}{2}=\frac{p^{2}-2 p}{8} .
$$

Substituting this value into the expression for $d\left(M_{p}, \mathbf{t}_{p / 2}\right)$ given above, the statement follows. 
Proof of Theorem 1.4. Suppose first that $p$ is even. Define $\xi_{p}$ by the contact surgery diagram of Figure 6. (In Figures 6 and 7, a coefficient $(+1)$ next to a Legendrian knot $K$ means that contact $(+1)$-surgery is performed along $K$, while no coefficient means contact (-1)-surgery). Our plan is to apply Theorem 1.2. Observe that this plan makes sense. In fact, it is easy to check that the proofs of Lemmas 4.1, 4.2 and Theorem 1.2 given in Section 4 apply without modifications to the contact surgery presentation of Figure 6 .

Turning the contact framings into smooth framings, a little Kirby calculus (as in [25, Figures 1 and 7]) shows that $\xi_{p}$ is a contact structure on $M_{p}$. Now we want to apply the formula from $[4]$ for the $d_{3}$-invariant of a contact structure defined by a contact $( \pm 1)$-surgery diagram. If $c \in H^{2}(X ; \mathbb{Z})$ denotes the 2 -cohomology class determined by the rotations numbers (see $[4]$ ), $\sigma(X)$ is the signature of $X$ and $b_{2}(X)$ the second Betti number, a simple computation yields

$$
\sigma(X)=1-p, \quad b_{2}(X)=p+3, \quad c^{2}=\frac{\left(p-\left(p^{2}-p-1\right)\right)^{2}}{p^{2}-p-1}-p(p-1) .
$$

Then, [4, Corollary 3.6] (where $b_{2}(X)$ should be plugged into the formula instead of the Euler characteristic $\chi(X)$ because the 3-dimensional invariant used in Heegaard Floer theory is shifted by $\frac{1}{2}$ ) gives

$$
d_{3}\left(\xi_{p}\right)=\frac{\left(p^{2}-2 p-1\right)^{2}}{4\left(p^{2}-p-1\right)}-\frac{1}{4}(p-1)^{2} .
$$

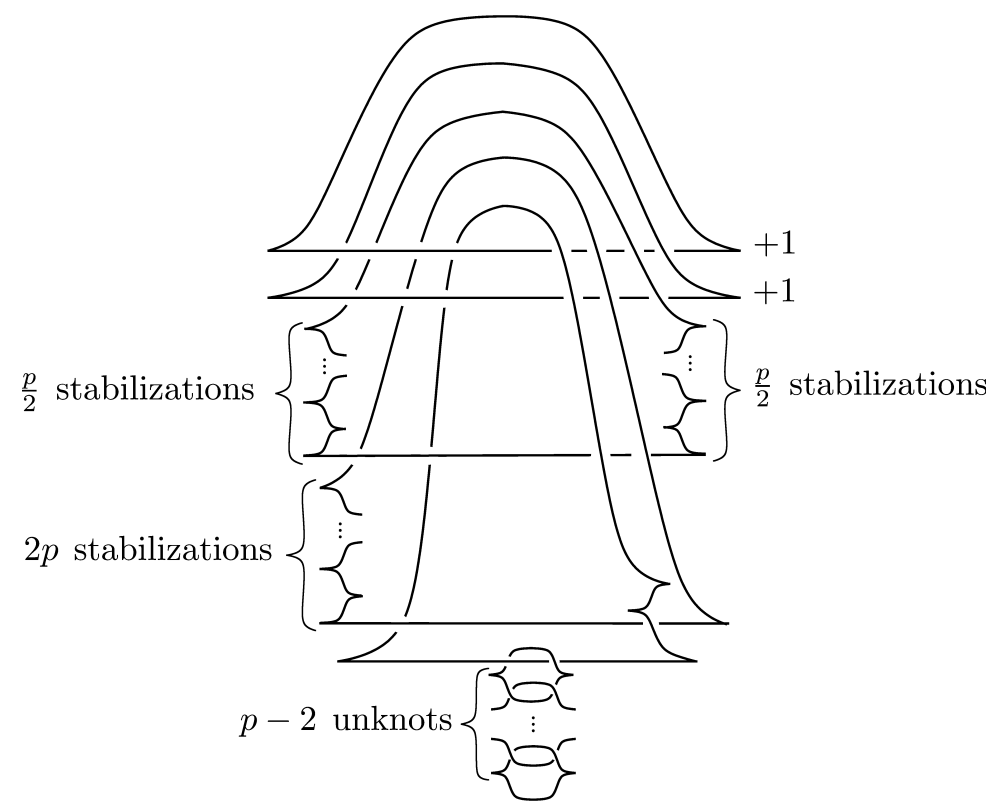

Figure 6. The contact structure $\xi_{p}$ for $p$ even. 
Following the blow-down procedure at the cohomological level, the verification that $\xi_{p}$ is a contact structure on the given 3-manifold $M_{p}$ also shows that for $p>2$

$$
\mathbf{t}_{\xi_{p}}=\mathbf{t}_{p / 2}
$$

(For $p=2$ the inequality $p<p^{2}-p-1$ fails to hold, and we have $\mathbf{t}_{\xi_{2}}=\mathbf{t}_{0}$.)

Therefore, by Lemma 5.4 we have

$$
d_{3}\left(\xi_{p}\right)=d\left(M_{p}, \mathbf{t}_{\xi_{p}}\right) .
$$

Using Theorem 1.2 we conclude that $c\left(M_{p}, \xi_{p}\right) \neq 0$, and hence $\xi_{p}$ is a tight contact structure on $M_{p}$.

We now verify the statement for $p$ odd. (This case was already treated in [25, Theorem 1.3].) Let $\xi_{p}$ denote the contact structure given by the contact surgery diagram of Figure 7. As in the previous case, we can apply Theorem 1.2. A computation as above shows that

$$
\sigma(X)=1-p, \quad b_{2}(X)=p+3, \quad c^{2}=-2 p
$$

and applying $[\mathbf{4}$, Corollary 3.6] we have

$$
d_{3}\left(\xi_{p}\right)=-\frac{1}{4}(p+1) \text { and } \quad \mathbf{t}_{\xi_{p}}=\mathbf{t}_{0} .
$$

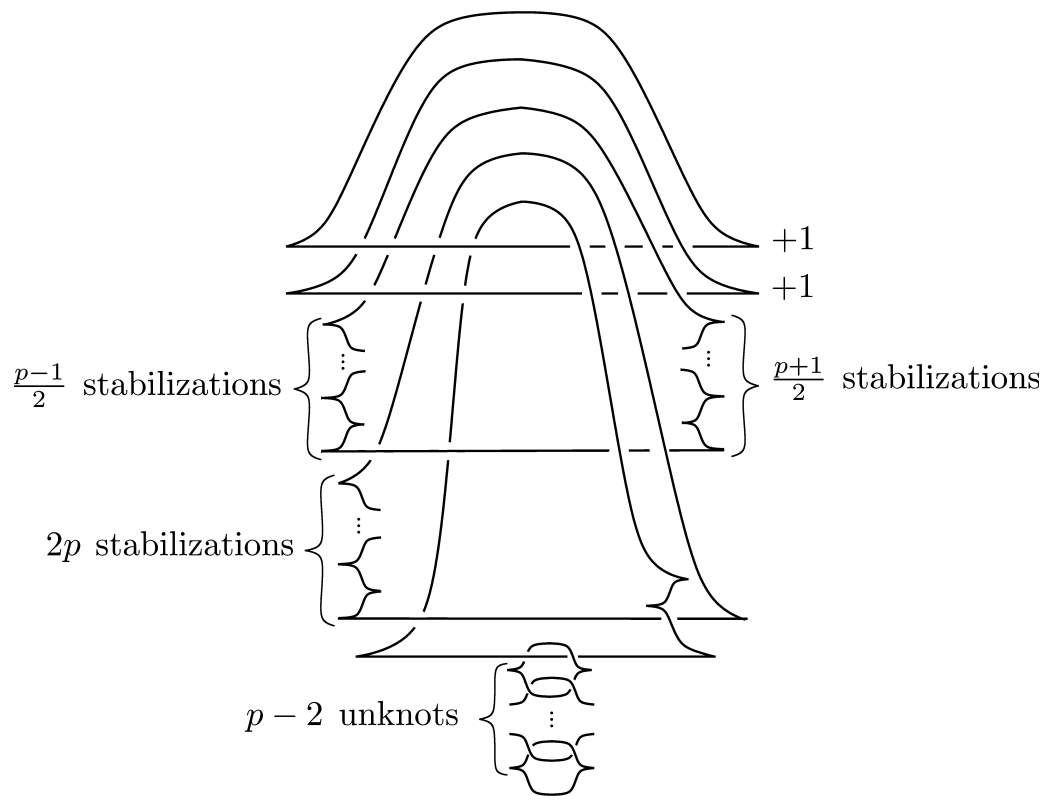

Figure 7. The contact structure $\xi_{p}$ for $p$ odd. 
Using Proposition 5.1 we see that

$$
d\left(M_{p}, \mathbf{t}_{\xi_{p}}\right)=-\frac{1}{4}(p+1)=d_{3}\left(\xi_{p}\right)
$$

which, in view of Theorem 1.2, implies that $\xi_{p}$ is tight and concludes the proof.

\section{Planar contact structures}

6.1. Surgery diagrams for contact structures with zero twisting. In this section, we show that if $\xi$ is a tight contact structure with maximal twisting equal to zero on the small Seifert fibered 3-manifold $M=M\left(-1 ; r_{1}, r_{2}, r_{3}\right)$, then $(M, \xi)$ is compatible with a planar open book decomposition of $M$, and we discuss some consequences. We start by establishing the fact that $(M, \xi)$ is given by one of the contact surgeries represented by Figure 3 .

Let $F_{i}(i=1,2,3)$ be the three singular fibers of the Seifert fibration on $M$. We first isotope $F_{i}$ so that they become Legendrian. Then, since $\xi$ has maximal twisting equal to zero, we can isotope the Seifert fibration further, so that there is a Legendrian regular fiber $L$ with contact framing equal to the framing induced on $L$ by the Seifert fibration.

Let $V_{i}$ be a standard neighborhood of $F_{i}, i=1,2,3$. Then $M \backslash\left(V_{1} \cup V_{2} \cup V_{3}\right)$ can be identified with $\Sigma \times S^{1}$ where $\Sigma$ is a pair-of-pants. An identification between $M \backslash\left(V_{1} \cup V_{2} \cup V_{3}\right)$ and $\Sigma \times S^{1}$ determines identifications of $-\partial(M \backslash$ $\left.V_{i}\right)$ with $\mathbb{R}^{2} / \mathbb{Z}^{2}$ so that $\left(\begin{array}{l}1 \\ 0\end{array}\right)$ is the direction of the section $\Sigma \times\{1\}$ and $\left(\begin{array}{l}0 \\ 1\end{array}\right)$ is the direction of the regular fibers. A standard application of convex surface theory (as in, e.g., [15]) shows that the singular fibers $F_{1}, F_{2}, F_{3}$ admit tubular neighborhoods $U_{1}, U_{2}, U_{3}$ with minimal convex boundaries such that $-\partial\left(M \backslash U_{i}\right)$ has infinite slope for $i=1,2,3$.

Proposition 6.1. Let $\xi$ be a tight contact structure with maximal twisting equal to zero on the small Seifert fibered 3 -manifold $M=M\left(-1 ; r_{1}, r_{2}, r_{3}\right)$. Then $(M, \xi)$ is given by one of the contact surgeries represented by Figure 3 .

Proof. Let $\Sigma$ be a pair of pants. Recall that a tight contact structure $\xi$ on $\Sigma \times S^{1}$ is appropriate if there is no contact embedding $\left(T^{2} \times I, \xi_{\pi}\right) \hookrightarrow$ $\left(\Sigma \times S^{1}, \xi\right)$, with $T^{2} \times\{0\}$ isotopic to a boundary component, where $\xi_{\pi}$ is a tight contact structure with convex boundary and twisting $\pi$ (see $[\mathbf{1 8}$, $\S 2.2 .1]$ for the definition of twisting). By the results of [15, Section 2], we are free to change the restriction $\left.\xi\right|_{M-\left(U_{1} \cup U_{2} \cup U_{3}\right)}$ without changing the isotopy class of $\xi$, as long as we replace $\left.\xi\right|_{M-\left(U_{1} \cup U_{2} \cup U_{3}\right)}$ by a tight, appropriate contact structure with minimal convex boundaries having the same infinite boundary slopes.

We claim that this contact structure can be chosen to be the contact complement $(C, \eta)$ of standard neighborhoods of the three Legendrian unknots 
$L_{1}, L_{2}, L_{3}$ in Figure 3 . In fact, the contact structure obtained by performing contact (-1)-surgeries along the knots $L_{1}, L_{2}, L_{3}$ is tight, due to the cancellation of $( \pm 1)$-surgeries of opposite signs along Legendrian push-offs [3]. Therefore $\eta$ is tight, and it is easy to check that $\eta$ is also appropriate because it extends to a tight contact structure on a closed 3-manifold obtained by filling the neighborhoods of $L_{1}, L_{2}$ and $L_{3}$. It is obvious that the boundary components of $(C, \eta)$ are minimal and convex. To check that the boundary components have infinite boundary slopes, it is enough to observe that there is a product structure $C \cong \Sigma \times S^{1}$ such that (i) a fiber $F$ of the projection $C \rightarrow \Sigma$ is Legendrian and has twisting number zero, and (ii) all the $L_{i}$ 's are Legendrian pushoffs of $F$.

6.2. Open book decompositions. According to a recent result of Giroux [16], isotopy classes of contact structures are in one-to-one correspondence with suitable equivalence classes of open book decompositions of the underlying 3-manifold; cf. also [7]. Recall that an open book decomposition $(Y, f)$ of a closed 3-manifold $Y$ amounts to a link $L \subset Y$ and a fibration $f: Y-L$ such that the closure of every fiber $f^{-1}(t)$ (called a page) provides a Seifert surface for $L$. The open book decomposition is called planar if the genus of the page is zero. We will also call planar any contact structure compatible with a planar open book decomposition. The following result relates planarity of a contact structure with dynamical properties of its Reeb vector fields.

Theorem 6.2 (Abbas-Cieliebak-Hofer [1]). If $\xi$ is a contact structure compatible with a planar open book decomposition then it satisfies the Weinstein conjecture, that is, any Reeb vector field of $\xi$ admits a periodic orbit.

Necessary conditions for a contact structure to be planar were found in $[\mathbf{8}$, 31]. We will prove Theorem 1.5 using Proposition 6.1. Before dwelving into the proof, we describe some consequences of Theorem 1.5, some of which immediately imply Corollaries 1.6, 1.7 and 1.8. First of all, we have the following.

Corollary 6.3. If $M=M\left(e_{0} ; r_{1}, r_{2}, r_{3}\right), e_{0} \geq-1$ and $M$ is an $L$-space, then each contact structure on $M$ is planar and therefore satisfies Weinstein's conjecture. $^{1}$

Proof. It is known that overtwisted contact structures are planar [8], and hence we can focus on tight structures only. For small Seifert fibered rational homology 3 -spheres with $e_{0} \geq 0$ it was shown by $\mathrm{Wu}[\mathbf{4 4}]$ that each tight contact structure has maximal twisting equal to zero, therefore Theorem 1.5 shows that on those manifolds every contact structure is planar.

\footnotetext{
${ }^{1}$ Added in proof: Cliff Taubes has recently posted a proof of the Weinstein conjecture for every contact 3 -manifold $[\mathbf{4 3}]$.
} 
Suppose now that $M$ is an $L$-space and $e_{0}(M)=-1$. By Theorem 1.1 the 3-manifold $M$ admits no transverse contact structures, and hence Theorem 1.3 shows that every tight contact structure on $M$ has maximal twisting equal to zero. Theorem 1.5 therefore implies that every tight contact structure on $M$ is planar.

Remark 6.4. In [42] Stephan Schönenberger proved that if $e_{0} \leq-3$ then every contact structure on $M=M\left(e_{0} ; r_{1}, r_{2}, r_{3}\right)$ is planar. Therefore, since in this case $M$ is always an $L$-space, the statement of Corollary 6.3 holds if $e_{0} \leq-3$. On the other hand, the Poincaré sphere admits a Seifert fibration with $e_{0}=-2$, is an $L$-space, but its unique Stein fillable contact structure is not planar because the intersection form of a filling is not diagonalizable $[\mathbf{8}, \mathbf{3 1}]$.

In view of Corollary 6.3, Theorem 1.1 implies the following characterization of $L$-spaces of the form $M\left(e_{0} ; r_{1}, r_{2}, r_{3}\right)$.

Corollary 6.5. The 3-manifold $M=M\left(e_{0} ; r_{1}, r_{2}, r_{3}\right)$ is an L-space if and only if one of the following holds:

- each contact structure on $M$ is planar;

- each contact structure on $-M$ is planar.

Proof. If $e_{0}(M) \geq 0$ then by Corollary 6.3 every contact structure on $M$ is planar. On the other hand, it is known that if $e_{0}(M) \geq 0$ or $e_{0}(M) \leq-3$ then $M$ is an $L$-space. Since $e_{0}(-M)=-3-e_{0}(M)$, this immediately implies the statement if $e_{0}(M) \geq 0$ or $e_{0}(M) \leq-3$. Suppose now that $M$ is an $L$-space and, up to changing its orientation, $e_{0}(M)=-1$. Then, by Corollary 6.3 every contact structure on $M$ is planar.

To finish the proof, we may assume without loss that $e_{0}(M)=-1$ and $M$ is not an $L$-space. According to Theorem 1.1 the manifold $M$ admits a taut foliation, which gives rise to a contact structure $\xi$ having a symplectic semi-filling. By $[\mathbf{5}, \mathbf{9}]$ one can use a symplectic cap to construct a symplectic filling of $(M, \xi)$ with $b_{2}^{+}>0$. Then, according to $[\mathbf{8}, \mathbf{3 1}] \xi$ is not planar.

Let $T_{p, n p+1}$ denote the positive $(p, n p+1)$-torus knot, and let

$$
M_{p, p n+1}=-S_{p^{2} n-p n-1}^{3}\left(T_{p, p n+1}\right) .
$$

Proposition 6.6 below shows that the non-planarity of the Stein fillable contact structure on the Poincaré sphere (cf. Remark 6.4) is a non-isolated phenomenon.

Proposition 6.6. For $p \geq 2$ and $n \geq 1$ the rational homology 3-sphere $M_{p, n p+1}$ is an L-space and it carries non-planar contact structures.

Proof. Since the slice genus of $T_{p, n p+1}$ is $p^{2} n-p n-1$, the fact that $M_{p, p n+1}$ is an $L$-space follows from [24, Proposition 4.1]. 
Let $W_{p, n}$ denote the canonical plumbing 4-manifold associated to $M_{p, n p+1}$ as in Section 2. Since all the weights of the plumbing are at most -2 (and all knots are unknots), $W_{p, n}$ supports Stein structures inducing tight contact structures on $M_{p, n}$. The proof of [8, Theorem 4.1] shows that if any of these structures is planar, then $W_{p, n}$ smoothly embeds in a connected sum of $\overline{\mathbb{C P}}^{2}$ 's. But the argument given in the proof of [25, Proposition 4.1] shows that the intersection form $Q_{W_{p, n}}$ does not embed into a diagonal lattice. Therefore, none of the contact structures filled by $W_{p, n}$ are planar.

Proof of Theorem 1.5. Let $\xi$ be a tight contact structure with twisting number equal to zero on $M\left(-1 ; r_{1}, r_{2}, r_{3}\right)$. By Proposition 6.1 this structure is obtained by performing a (possibly rational) contact surgery along the fivecomponent Legendrian link $\mathcal{L}$ of Figure 2 (for $k=3$ ). According to the algorithm outlined in Section $2, \xi$ is obtained by contact $( \pm 1)$-surgery on a Legendrian link $\tilde{\mathcal{L}}$ obtained from $\mathcal{L}$ by successively taking pushoffs and Legendrian stabilizations of (some of) its components. It is well known that performing contact $( \pm 1)$-surgery on a Legendrian knot which sits on a page of a compatible open book with contact framing equal to the page framing yields an open book of the same genus compatible with the resulting contact structure. Therefore, it suffices to show that $\tilde{\mathcal{L}}$ sits on a union of pages of a planar open book for $S^{3}$ compatible with the standard contact structure. This can be proved by an argument very similar to the one used in $[\mathbf{4 2}]$ to prove that each contact structure on a lens space is planar. From now on, we refer to [7] for standard facts on contact structures and their compatible open books. Start with the open book decomposition of $S^{3}$ compatible with the standard contact structure whose page is an annulus and whose binding is a Hopf link. By applying the Legendrian realization principle, a Legendrian unknot with maximal Thurston-Bennequin invariant together with four of its pushoffs can be realized on five distinct pages of this open book, so that contact framings and page framings coincide. Up to positively stabilizing the open book in the sense of Giroux, we can realize in the same way any Legendrian stabilizations of these unknots on distinct pages of a planar open book compatible with the standard contact structure on $S^{3}$. Any number of pushoffs of the stabilized knots can then be realized on distinct pages, any further Legendrian stabilization can be realized on further planar stabilizations of the open book, and so on. This construction clearly establishes what we need.

\section{References}

[1] C. Abbas, K. Cieliebak and H. Hofer, The Weinstein conjecture for planar contact structures in dimension three, Comment. Math. Helv. 80 (2005), 771-793.

[2] G. Burde and H. Zieschang, Knots, de Gruyter Studies in Mathematics, 5, Walter de Gruyter \& Co., Berlin, 1985. 
[3] F. Ding and H. Geiges, A Legendrian surgery presentation of contact 3-manifolds, Math. Proc. Cambridge Philos. Soc. 136 (2004), 583-598.

[4] F. Ding, H. Geiges and A. Stipsicz, Surgery diagrams for contact 3-manifolds, Turkish J. Math. 28 (2004), 41-74.

[5] Y. Eliashberg, A few remarks about symplectic filling, Geom. Topol. 8 (2004), 277-293.

[6] Y. Eliashberg and W. Thurston, Confoliations, University Lecture Series, 13, American Mathematical Society, Providence, RI, 1998.

[7] J. Etnyre, Lectures on open book decompositions and contact structures, Floer homology, gauge theory, and low-dimensional topology, Clay Mathematics Proceedings, 5, American Mathematical Society, Providence, RI, 2006, 103-141.

[8] J. Etnyre, Planar open book decompositions and contact structures, Int. Math. Res. Not. 2004 (79) (2004), 4255-4267.

[9] J. Etnyre, On symplectic fillings, Algebr. Geom. Topol. 4 (2004), 73-80.

[10] J. Etnyre and K. Honda, On the nonexistence of tight contact structures, Ann. of Math. 153 (2001), 749-766.

[11] J. Etnyre and K. Honda, Tight contact structures with no symplectic fillings, Invent. Math. 148 (2002), 609-626.

[12] P. Ghiggini, Ozsváth-Szabó invariants and fillability of contact structures, Math. Z. 253 (2006), 159-175.

[13] P. Ghiggini, On tight contact structures with negative maximal twisting on small Seifert manifolds, arXiv:0707.4494v1.

[14] P. Ghiggini, P. Lisca and A. Stipsicz, Classification of tight contact structures on small Seifert 3-manifolds with $e_{0} \geq 0$, Proc. Amer. Math. Soc. 134 (2006), 909-916.

[15] P. Ghiggini, P. Lisca and A. Stipsicz, Tight contact structures on some small Seifert fibered 3-manifolds, arxiv:math.SG/0509714, Amer. J. Math. 129 (5) (2007), 1403-1447.

[16] E. Giroux, Géométrie de contact: de la dimension trois vers les dimensions supérieures, Proceedings of the International Congress of Mathematicians, Vol. II (Beijing, 2002), Higher Ed. Press, Beijing, 2002, 405-414.

[17] R. Gompf, Handlebody constructions of Stein surfaces, Ann. of Math. 148 (1998), 619-693.

[18] K. Honda, On the classification of tight contact structures, I., Geom. Topol. 4 (2000), 309-368.

[19] K. Honda, Confoliations transverse to vector fields, Preprint, (1998), preliminary version, http://almaak.usc.edu/ ${ }^{\sim}$ khonda/research.html.

[20] M. Jankins and W. Neumann, Rotation numbers of products of circle homeomorphisms, Math. Ann. 271 (1985), 381-400.

[21] P. Lisca and G. Matić, Transverse contact structures on Seifert 3-manifolds, Algebr. Geom. Topol. 4 (2004), 1125-1144.

[22] P. Lisca and A. Stipsicz, An infinite family of tight, not semi-fillable contact 3-manifolds, Geom. Topol. 7 (2003), 1055-1073.

[23] P. Lisca and A. Stipsicz, Seifert fibered contact three-manifolds via surgery, Alg. Geom. Topol. 4 (2004), 199-217. 
[24] P. Lisca and A. Stipsicz, Ozsváth-Szabó invariants and tight contact three-manifolds. I, Geom. Topol. 8 (2004), 925-945.

[25] P. Lisca and A. Stipsicz, Ozsváth-Szabó invariants and tight contact three-manifolds. II., J. Differential Geom. 75 (1) (2007), 109-141.

[26] J. McCarthy and J. Wolfson, Symplectic gluing along hypersurfaces and resolution of isolated orbifold singularities, Invent. Math. 119 (1995), 129-154.

[27] R. Naimi, Foliations transverse to fibers of Seifert manifolds, Comment. Math. Helv. 69 (1994), 155-162.

[28] A. Némethi, On the Ozsváth-Szabó invariant of negative definite plumbed 3-manifolds, Geom. Topol. 9 (2005), 991-1042.

[29] W. Neumann and F. Raymond, Seifert manifolds, plumbing, $\mu$-invariant and orientation reversing maps, Algebraic and geometric topology (Proc. Sympos., Univ. California, Santa Barbara, Calif., 1977), Lecture Notes in Mathematics 664, Springer, Berlin, 1978, 163-196.

[30] P. Orlik, Seifert manifolds, Lecture Notes in Mathematics, 291, Springer-Verlag, Berlin-New York, 1972.

[31] P. Ozsváth, A. Stipsicz and Z. Szabó, Planar open books and Floer homology, Int. Math. Res. Not. 2005 (54) (2005), 3385-3401.

[32] P. Ozsváth and Z. Szabó, Absolutely graded Floer homologies and intersection forms for four-manifolds with boundary, Adv. Math. 173 (2003), 179-261.

[33] P. Ozsváth and Z. Szabó, On the Floer homology of plumbed three-manifolds, Geom. Topol. 7 (2003), 185-224.

[34] P. Ozsváth and Z. Szabó, Heegaard diagrams and holomorphic disks, Different faces of geometry, International Mathematical Series (NY), 3, Kluwer/Plenum, New York, 2004, 301-348.

[35] P. Ozsváth and Z. Szabó, Holomorphic disks and genus bounds, Geom. Topol. 8 (2004), 311-334.

[36] P. Ozsváth and Z. Szabó, Holomorphic disks and topological invariants for closed three-manifolds, Ann. of Math. 159 (2004), 1027-1158.

[37] P. Ozsváth and Z. Szabó, Holomorphic disks and three-manifold invariants: properties and applications, Ann. of Math. 159 (2004), 1159-1245.

[38] P. Ozsváth and Z. Szabó, Heegaard Floer homologies and contact structures, Duke Math. J. 129 (1) (2005), 39-61.

[39] P. Ozsváth and Z. Szabó, On knot Floer homology and lens space surgery, Topology 44 (6) (2005), 1281-1300.

[40] P. Ozsváth and Z. Szabó, Holomorphic triangles and invariants of smooth 4-manifolds, Adv. Math. 202 (2) (2006), 326-400.

[41] P. Ozsváth and Z. Szabó, Knot Floer homology and rational surgeries, arxiv:math.GT/0504404.

[42] S. Schönenberger, Planar open books and symplectic fillings, PhD thesis, University of Pennsylvania, 2005.

[43] C. Taubes, The Seiberg-Witten equations and the Weinstein conjecture, arXiv:math/0611007.

[44] H. Wu, Legendrian vertical circles in small Seifert spaces, Comm. Contemp. Math. 8 (2) (2006), 219-246. 
Dipartimento di Matematica L. Tonelli

Università DI PISA

Largo Bruno Pontecorvo 5

I-56127 PisA, ITALY

E-mail address: lisca@dm.unipi.it

RÉNyi Institute of Mathematics

Hungarian ACADEmy of Sciences

H-1053 BUDAPEST

ReÁltanoda utca 13-15, Hungary

Institute for Advanced Study

Princeton, NJ

E-mail address: stipsicz@math-inst.hu

Received 11/07/2005, accepted 08/02/2007

The authors are grateful to Stefan Friedl for suggesting the neat argument used in the proof of Proposition 5.3. The first author was partially supported by MURST. The second author was partially supported by OTKA T049449. 